\title{
A nonsingular solution of the edge dislocation in the gauge theory of dislocations
}

\author{
Markus Lazar \\ Max-Planck-Institute for Mathematics in the Sciences, \\ Inselstr. 22-26, D-04103 Leipzig, Germany \\ E-mail: lazar@mis.mpg.de
}

July 17, 2018

\begin{abstract}
A (linear) nonsingular solution for the edge dislocation in the translational gauge theory of defects is presented. The stress function method is used and a modified stress function is obtained. All field quantities are globally defined and the solution agrees with the classical solution for the edge dislocation in the far field. The components of the stress, strain, distortion and displacement field are also defined in the dislocation core region and they have no singularity there. The dislocation density, moment and couple stress for an edge dislocation are calculated. The solutions for the stress and strain field obtained here are in agreement with those obtained by Gutkin and Aifantis through an analysis of the edge dislocation in the strain gradient elasticity. Additionally, the relation between the gauge theory and Eringen's so-called nonlocal theory of dislocations is given.
\end{abstract}

Keywords: elastoplasticity, dislocation theory, gauge theory of defects, strain gradient, nonlocal elasticity

PACS: $61.72 . L k, 62.20 .-\mathrm{x}, 81.40 . \mathrm{Jj}$

MPI-MIS $65 / 2002$

cond-mat/0208360

\section{Introduction}

The gauge theory of dislocations [1-22] is a very attractive field of research. The theory of dislocations is in these theories considered as a translational gauge theory (T(3)-gauge theory). An important goal is to find modified solutions of the stress and strain field of screw and edge dislocations in this framework because the "classical" elasticity of dislocations contains singularities at the dislocation line. Therefore, the classical solutions cannot be applied within the dislocation core region. This is unfortunate since the core region is the most important from the point of dislocation and failure mechanics. The difference in these gauge models is the form of the $T(3)$-gauge Lagrangian. Edelen et al. $[6-8,10]$ used the simplest form quadratic in the $T(3)$-gauge field strength. In $[1-4,13]$ the gauge Lagrangian was proposed in the three-dimensional Hilbert-Einstein form. The most general dislocation gauge Lagrangian for an isotropic and anisotropic material was proposed in $[1,2]$. 
A correct gauge theoretical solution of the stress field of a screw dislocation is given in $[2-4,9,10]$. This solution has no singularity at the dislocation line and the far field stress is that of a classical screw dislocation.

Up to now, the situation is less satisfactory for the edge dislocation. In the Edelen model [6-8] the far field of the stress $\sigma_{z z}$ is different from the standard one. In fact, the far field stress $\sigma_{z z}$ is equal to $\nu^{-1}\left(\sigma_{x x}+\sigma_{y y}\right)$ instead of $\nu\left(\sigma_{x x}+\sigma_{y y}\right)$ (see also [4]). Unfortunately, no explicit gauge solution for the edge dislocation was given in [6-8]. Moreover, the structure of the gauge Lagrangian used by Edelen et al. requires, in principle, an asymmetric force stress [2]. Malyshev [4] used the HilbertEinstein Lagrangian as a gauge Lagrangian and found the stress field of a straight edge dislocation with a modified asymptotic behaviour which differs from the classical edge dislocation. In fact, the stress field has no singularity at the dislocation line and has an oscillatory behaviour with slowly decreasing amplitude far from the dislocation core. His solution can be considered as a complicated gauge defect with modified asymptotics instead of an elementary defect [4].

On the other hand, there are other non-standard continuum models of defects, e.g., the Peierls-Nabarro model [23-26], the nonlocal continuum model [27-31] and the strain gradient elasticity [32-38]. The force stresses calculated in these theories are symmetric even in the core. In the case of a screw dislocation, the solution of the force stresses in nonlocal elasticity $[28,30,31]$ and in gradient elasticity $[37,38]$ agrees with the gauge theoretical one $[2-4,9,10]$. Additionally, the strain fields calculated in gradient theory $[35,37,38]$ and in gauge theory $[2,3,9,10]$ coincide. In the case of an edge dislocation, the solution in nonlocal elasticity was given in an integral form by means of a special two-dimensional nonlocal kernel [30]. Using another twodimensional nonlocal kernel Eringen [27] gave a different solution of the straight edge dislocation. The dislocation stress field in gradient elasticity [36-38] was obtained in a closed analytical form by using the Fourier transform method, it is equal to zero at the dislocation line and beyond the dislocation core the classical and the gradient solutions coincide. In a quasi-continuum model [31,39-42] a modified stress field of an edge and a screw dislocation was obtained, also. In this approach the stress field has no divergence at the dislocation line and at large distances it contains a decreasing oscillatory contribution. Dislocations have also been considered in a Cosserat media (see, e.g., [43-45]). The force stresses of dislocations calculated within the Cosserat theory are, in general, asymmetric. The solutions of edge and screw dislocations [46-52] found for the elastic strain and stress fields differ from the classical solutions but still posses singularities at the dislocation line.

A common feature of gauge theory, nonlocal elasticity, strain gradient theory and theory of Cosserat media of defects is that a characteristic length scale enters the constitutive laws. In the gauge theory of defects and in the theory of Cosserat and multipolar media this length is a material property which carries with it all of the difference between solutions with or without moment stresses. Its influence might be become important as dimensions of the body or wavelengths diminish to the order of the characteristic inner length. By means of this characteristic length scale it is possible to define the dislocation core radius in a straightforward manner [2].

The question arises: Can we find a gauge theoretical solution for the edge dislocation which has the correct far field stress and is related to any solution of another framework? The aim of this paper is to find an answer to this question, that means to construct a correct gauge theoretical solution of a straight edge dislocation. Additionally, the relation to the nonlocal theory and strain gradient elasticity of an edge dislocation will be given. Here we restrict our considerations to the case of linear dislocation theory. 


\section{The translational gauge invariant formulation of dislocation theory}

For the undeformed reference state of the body the indices $i=1,2,3$ are used. The final coordinates of the deformed state are labelled by the indices $a=1,2,3$. In presence of defects the deformed state of the material is characterized by nonholonomic coordinates. In linear theory we may use $\delta_{a}^{i}, \delta_{i j}$ and $\delta_{a b}$ to raise and lower the indices.

In elasticity theory the state of an elastic body is not changed under a rigid translation and rotation (global gauge transformations). In elasticity the (compatible) distortion is given as a proper gradient

$$
\beta^{a}{ }_{i}=\partial_{i} u^{a} .
$$

The distortion tensor is dimensionless. The basic idea of gauge theory of defects is now that the distortion has to be invariant not only under the global gauge transformation but also under if the gauge group is applied locally. In this way, one obtains the incompatible distortion in the framework of translational gauge theory of dislocations as follows

$$
\beta_{i}^{a}=\partial_{i} u^{a}+\phi_{i}^{a} .
$$

It is locally translational invariant (gauge invariant) due to

$$
u^{a} \longrightarrow u^{a}+\tau^{a}(x), \quad \phi_{i}^{a} \longrightarrow \phi_{i}^{a}-\partial_{i} \tau^{a}(x),
$$

where $\tau^{a}(x)$ are local translations [2]. Here $u^{a}$ is the displacement field from the undeformed to the deformed configuration and $\phi^{a}{ }_{i}$ is the proper incompatible part of the distortion. The incompatible distortion (2.2) can be understood as the (minimal) replacement of the compatible distortion (2.1) in the $T(3)$-gauge theory

$$
\partial_{i} u^{a} \longrightarrow \partial_{i} u^{a}+\phi^{a}{ }_{i} .
$$

Then, the (incompatible) strain tensor turns out to be ${ }^{1}$

$$
E_{i j}=\frac{1}{2}\left(\delta_{a i} \beta^{a}{ }_{j}+\delta_{a j} \beta_{i}^{a}\right) \equiv \beta_{(i j)} .
$$

The skew-symmetric part of the distortion defines the elastic rotation tensor

$$
\omega_{i j}=\frac{1}{2}\left(\delta_{a i} \beta_{j}^{a}-\delta_{a j} \beta_{i}^{a}\right) \equiv \beta_{[i j]},
$$

and the elastic rotation vector

$$
\omega_{i} \equiv-\frac{1}{2} \epsilon_{i}^{j k} \delta_{a j} \beta_{k}^{a} .
$$

The elastic strain energy of an isotropic media is defined by

$$
W=\frac{1}{2} \mu\left(\delta^{i k} \delta^{j l}+\delta^{i l} \delta^{j k}+\frac{2 \nu}{1-2 \nu} \delta^{i j} \delta^{k l}\right) E_{i j} E_{k l},
$$

where $\mu$ is the shear modulus and $\nu$ is Poisson's ratio. It is now invariant under local translations. Obviously, no elastic rotation enters the relation (2.8) and, therefore,

\footnotetext{
${ }^{1}$ We will be using the notations $A_{(i j)} \equiv \frac{1}{2}\left(A_{i j}+A_{j i}\right)$ and $A_{[i j]} \equiv \frac{1}{2}\left(A_{i j}-A_{j i}\right)$.
} 
the elastic rotation has no elastic response quantity. The force stress can be derived from the strain energy

$$
\sigma^{i j}=\frac{\partial W}{\partial E_{i j}}=2 \mu\left(E^{i j}+\frac{\nu}{1-2 \nu} \delta^{i j} E_{k}^{k}\right) .
$$

It is the elastic response quantity to the strain tensor. Formula (2.9) expresses the (incompatible) stress-strain relation (generalized Hooke's law).

It is well known that nontrivial traction boundary problems in the variational formulation of the gauge theory of defects can be formulated by means of a so-called null Lagrangian $[8,10,53]$. When the null Lagrangian is added to the Lagrangian of elasticity, it does not change the Euler-Lagrange equation (force equilibrium) because the associated Euler-Lagrange equation, $\partial_{j} \stackrel{\circ}{\sigma}_{a}^{j}=0$, is identically satisfied. After minimal replacement, $\partial_{i} u^{a} \rightarrow \beta_{i}^{a}$, the null Lagrangian

$$
W_{\mathrm{bg}}=\partial_{j}\left(\stackrel{\circ}{\sigma}_{a}^{j} u^{a}\right)=\left(\partial_{j} \stackrel{\circ}{\sigma}_{a}^{j}\right) u^{a}+\stackrel{\circ}{\sigma}_{a}^{j} \partial_{j} u^{a} \longrightarrow \stackrel{\circ}{\sigma}_{a}^{j} \beta_{j}^{a},
$$

give rise to a background stress tensor $\stackrel{\circ}{\sigma}_{a}^{j}$ which can be considered as the nucleation field in the gauge theory of defects [10].

As usual we use the translational field strength (torsion) to define the dislocation density tensor $[1-3,16,17,54-57]$

$$
T^{a}{ }_{i j}=\partial_{i} \beta_{j}^{a}-\partial_{j} \beta_{i}^{a},
$$

which has the dimension of an inverse length. The dislocation density is a fundamental quantity in plasticity because the dislocation is the elementary carrier of plasticity. This field is a physical state quantity which, at least in principle, can be measured without knowing anything about the history of the body. It is possible with the help of high resolution transmission electron microscopes (HRTEM) to see single dislocations with their core configuration in crystals. Since dislocations change the energy of the body (crystal), they should appear in the total Lagrangian. The dislocation Lagrangian, which we use, contains only the translational gauge field strength and is given by

$$
\mathcal{L}_{\text {disl }}=-\frac{1}{4} T^{a}{ }_{i j} H_{a}{ }^{i j}
$$

where the moment stress $H_{a}{ }^{i j}$ is the response quantity to dislocation density. This means, that at all positions where the dislocation density is non zero, also localized moment stresses are present. It is convenient to perform an irreducible decomposition of the torsion, from which we can construct the most general isotropic constitutive law between the dislocation density and the moment stress in the following way $[1,2]$

$$
H_{a i j}=\sum_{I=1}^{3} a_{I}{ }^{(I)} T_{a i j} .
$$

Here $a_{1}, a_{2}$ and $a_{3}$ are new material coefficients. They have the dimension of a force. The irreducible decomposition of the torsion under the rotation group $S O(3)$ reads

$$
T_{a i j}={ }^{(1)} T_{a i j}+{ }^{(2)} T_{a i j}+{ }^{(3)} T_{a i j} .
$$

The tensor, the trace and the axial tensor pieces are defined by (see also $[2,58]$ )

$$
\begin{aligned}
{ }^{(1)} T_{a i j}=T_{a i j}-{ }^{(2)} T_{a i j}-{ }^{(3)} T_{a i j} & & \text { (tentor), } \\
{ }^{(2)} T_{a i j}:=\frac{1}{2}\left(\delta_{a i} T_{l j}^{l}+\delta_{a j} T_{i l}^{l}\right) & & \text { (trator), } \\
{ }^{(3)} T_{a i j}:=\frac{1}{3}\left(T_{a i j}+T_{i j a}+T_{j a i}\right) & & \text { (axitor). }
\end{aligned}
$$


Then, the moment stress tensor reads

$$
H_{a i j}=-2 \frac{\partial \mathcal{L}_{\mathrm{disl}}}{\partial T^{a i j}}=c_{1} T_{a i j}+c_{2}\left(T_{i j a}+T_{j a i}\right)+c_{3}\left(\delta_{a i} T_{l j}^{l}+\delta_{a j} T^{l}{ }_{i l}\right),
$$

with the abbreviations

$$
c_{1}:=\frac{1}{3}\left(2 a_{1}+a_{3}\right), \quad c_{2}:=\frac{1}{3}\left(a_{3}-a_{1}\right), \quad c_{3}:=\frac{1}{2}\left(a_{2}-a_{1}\right) .
$$

The Euler-Lagrange equations for the Lagrangian $\mathcal{L}=\mathcal{L}_{\text {disl }}-W+W_{\text {bg }}$ turn out to be $[2]$

$$
\begin{array}{ll}
\frac{\delta \mathcal{L}}{\delta u^{a}} \equiv \partial_{j} \sigma_{a}{ }^{j}=0 & \text { (force equilibrium) }, \\
\frac{\delta \mathcal{L}}{\delta \phi^{a}{ }_{j}} \equiv \partial_{i} H_{a}{ }^{i j}=\widehat{\sigma}_{a}{ }^{j} &
\end{array}
$$

with the so-called effective stress tensor

$$
\widehat{\sigma}_{a}^{j}:=\sigma_{a}^{j}-\stackrel{\circ}{\sigma}_{a}^{j},
$$

which drives the dislocation fields. The field equations (2.20) and (2.21) determine the fields $\beta_{j}^{a}$ and $T_{i j}^{a}$ which represent the geometric (or kinematic) degrees of freedom. Note that in our approach the dislocation density is not given, a priori, as delta function. In this field theoretical context, we are able to calculate even the dislocation core. The static responses are the fields $\sigma_{a}^{j}$ and $H_{a}^{i j}$.

The L.H.S. of (2.21) can be written in terms of the distortion tensor

$$
\begin{aligned}
\partial^{i} H_{a i j}=c_{1}\left(\Delta \beta_{a j}-\partial^{i} \partial_{j} \beta_{a i}\right) & +c_{2}\left(\partial^{i} \partial_{j} \beta_{i a}-\partial_{a} \partial^{i} \beta_{i j}+\partial_{a} \partial^{i} \beta_{j i}-\Delta \beta_{j a}\right) \\
& +c_{3}\left(\partial_{a} \partial^{i} \beta_{i j}-\partial_{a} \partial_{j} \beta^{i}{ }_{i}+\delta_{a j} \Delta \beta^{i}{ }_{i}-\delta_{a j} \partial^{i} \partial^{k} \beta_{i k}\right) .
\end{aligned}
$$

In the general case the gauge theory of dislocations for isotropic materials contains three new material coefficients which can be used to define three internal characteristic lengths. In order to obtain only one coefficient from the three ones we need a suitable choice for the moment stress tensor $H_{a i j}$ and the coefficients $a_{1}, a_{2}$ and $a_{3}$, respectively. By means of such a choice we want to find a gauge theoretical solution of symmetric force stresses for the edge dislocation. If we use the choice ${ }^{2}$

$$
a_{2}=\frac{1+\nu}{1-\nu} a_{1}, \quad a_{3}=-\frac{a_{1}}{2}, \quad \Longrightarrow \quad c_{1}=\frac{a_{1}}{2}, \quad c_{2}=-\frac{a_{1}}{2}, \quad c_{3}=\frac{\nu}{1-\nu} a_{1},
$$

the localized moment stress tensor reads

$$
H_{a i j}=\frac{a_{1}}{2}\left(T_{a i j}-T_{i j a}-T_{j a i}+\frac{2 \nu}{1-\nu}\left(\delta_{a i} T_{l j}^{l}+\delta_{a j} T_{i l}^{l}\right)\right) .
$$

With the relations $H_{a k}=\frac{1}{2} \epsilon_{i j k} H_{a}^{i j}$ and $T_{i j}^{a}=\epsilon_{i j k} \alpha^{a k}$, and by using the conventional (microscopic) dislocation density tensor $\alpha^{a i}=\epsilon^{i j k} \partial_{j} \beta_{k}^{a}$ and the Nye [59] tensor ${ }^{3} \kappa_{a j}$

$$
\kappa_{a j}=\alpha_{j a}-\frac{1}{2} \delta_{a j} \alpha_{k}^{k}, \quad \alpha_{a j}=\kappa_{j a}-\delta_{a j} \kappa_{k}^{k}
$$

\footnotetext{
${ }^{2}$ Note that Malyshev [4] used the so-called Einstein choice $a_{2}=-a_{1}$ and $a_{3}=-\frac{a_{1}}{2}$ for an (modified) edge dislocation. By applying the stress function method he found a gauge theoretical solution for the edge dislocation in terms of the Bessel and Neumann functions and the modified Bessel functions. His solution of an (modified) edge dislocation has no singularity at $r=0$ but differs asymptotically from the classical edge dislocation. In detail the symmetric stress field, the closure failure and the dislocation density show a decaying behaviour with oscillations. Additionally, his solution violates in the dislocation core region the plane-strain condition $E_{z z}=0$.

${ }^{3}$ Here the tensor $\kappa_{a j}$ is a microscopic quantity.
} 
we obtain from (2.18) the relation

$$
H_{a j}=a_{1}\left(\alpha_{a j}-\frac{1}{2} \delta_{a j} \alpha_{k}^{k}+\frac{2 \nu}{1-\nu} \alpha_{[a j]}\right)=a_{1}\left(\kappa_{j a}+\frac{2 \nu}{1-\nu} \kappa_{[j a]}\right) .
$$

Alternatively, the dislocation Lagrangian (2.12) can be written in the form

$$
\mathcal{L}_{\text {disl }}=-\frac{1}{2} \alpha^{a i} H_{a i}
$$

By using (2.27) the L.H.S. of (2.21) reads

$$
\begin{aligned}
\partial^{i} H_{a i j}=a_{1} & \left\{\Delta \beta_{(a j)}-\partial^{i} \partial_{j} \beta_{(a i)}+\frac{1}{1-\nu} \partial_{a} \partial^{i} \beta_{[i j]}\right. \\
& \left.-\frac{\nu}{1-\nu}\left(\left(\partial_{a} \partial_{j}-\delta_{a j} \Delta\right) \beta_{i}^{i}+\delta_{a j} \partial^{i} \partial^{k} \beta_{i k}-\partial_{a} \partial^{i} \beta_{(i j)}\right)\right\} .
\end{aligned}
$$

Obviously, the strain and the rotation tensor appear in (2.29) because the distortion is not symmetric. It is reminded that in the so-called Hilbert-Einstein choice only the strain tensor enters the moment equilibrium (see $[2,3]$ ).

We now define the rotation gradient ${ }^{4}$

$$
k^{a}{ }_{i j}=\partial^{a} \beta_{[i j]} .
$$

It has the dimension of reciprocal of length. The rotation gradient describes a "latticecurvature". It contains two pieces. To see this we rewrite (2.30)

$$
k_{i j k}=\left(\partial_{k} E_{i j}-\partial_{j} E_{i k}\right)+\frac{1}{2}\left(T_{i j k}-T_{j k i}-T_{k i j}\right) .
$$

The first one is caused by local (incompatible) elastic strain and the other one by dislocations (contortion) [57]. The second piece may be identified with the plastic "lattice-curvature" which cannot be realized without dislocations. We add the following gauge invariant Lagrangian which looks like a rotation gradient Lagrangian of a Cosserat medium (see, e.g., [62])

$$
\mathcal{L}_{\text {grad }}=-\frac{1}{2} k^{a}{ }_{i j} \mu_{a}^{i j}
$$

The response quantity to the rotation gradient is a so-called "Cosserat" couple stress for an isotropic material ${ }^{5}$ (see, e.g., [62])

$$
\mu_{a[i j]}=-\frac{\partial \mathcal{L}_{\mathrm{grad}}}{\partial k^{a[i j]}}=d_{1} k_{a[i j]}+d_{2}\left(k_{i[j a]}+k_{j[a i]}\right)+d_{3}\left(\delta_{a i} k_{[l j]}^{l}+\delta_{a j} k_{[i l]}^{l}\right) .
$$

This constitutive relation uses the three rotation gradients, which are invariant under the $S O(3)$, and is analogous to the invariant constitutive relation for the torsion (2.18). We find

$$
\partial^{i} \mu_{i[a j]}=d_{1} \Delta \beta_{[a j]}+\left(d_{2}-d_{3}\right)\left(\partial^{i} \partial_{j} \beta_{[i a]}-\partial^{i} \partial_{a} \beta_{[i j]}\right) .
$$

Another gauge invariant Lagrangian, which we add, is given in terms of the torsion and the rotation gradient

$$
\mathcal{L}_{\text {grad-disl }}=-\frac{1}{2} d_{0} k^{a}{ }_{i j} T_{a}{ }^{i j} .
$$

\footnotetext{
${ }^{4}$ DeWit $[60,61]$ called such a rotation gradient elastic bend-twist.

${ }^{5}$ The relations between the constants in (2.33) and those in [62] are: $d_{1}=2 \alpha_{2}, d_{2}=\alpha_{3}, d_{3}=-\alpha_{1}$.
} 
Equation (2.35) may be considered as a kind of an interaction Lagrangian between dislocations and the "lattice-curvature". We define the response quantity

$$
\lambda_{a i j}=-\frac{\partial \mathcal{L}_{\text {grad-disl }}}{\partial\left(\partial^{i} \beta^{a j}\right)}=d_{0}\left(k_{a i j}+\frac{1}{2} T_{i a j}\right),
$$

with

$$
\partial^{i} \lambda_{a i j}=d_{0} \partial^{i}\left(\partial_{a} \beta_{[i j]}+\frac{1}{2}\left(\partial_{a} \beta_{i j}-\partial_{j} \beta_{i a}\right)\right) .
$$

The coefficients $d_{0}, d_{1}, d_{2}$ and $d_{3}$ have the dimensions of forces.

If we use the Lagrangian $\mathcal{L}=\mathcal{L}_{\text {disl }}+\mathcal{L}_{\text {grad }}+\mathcal{L}_{\text {grad-disl }}-W+W_{\text {bg }}$, the moment equilibrium reads

$$
\partial^{i}\left(H_{a i j}+\lambda_{a i j}+\mu_{i[a j]}\right)=\widehat{\sigma}_{a j},
$$

which can be decomposed into its symmetric and antisymmetric part. For the symmetric part of the moment equilibrium we obtain the equation

$$
\partial^{i}\left(H_{(a j) i}+\lambda_{(a j) i}\right)+\widehat{\sigma}_{(a j)}=0 .
$$

By using the choice of the coefficient

$$
d_{0}=-\frac{a_{1}}{(1-\nu)}
$$

formula (2.39) can be rewritten in terms of the strain tensor, only, according to

$a_{1}\left\{\Delta E_{i j}-\partial^{k} \partial_{(i} E_{j) k}-\frac{\nu}{1-\nu}\left(\left(\partial_{i} \partial_{j}-\delta_{i j} \Delta\right) E_{k}^{k}+\delta_{i j} \partial^{k} \partial^{l} E_{k l}-\partial^{k} \partial_{(i} E_{j) k}\right)\right\}=\widehat{\sigma}_{i j}$.

This equation can be interpreted as the (static) field equation in the elastoplastic theory of dislocations. It is derived from the combination of several Lagrangians Eqs. (2.12), (2.32), (2.35) - and the consideration of the null Lagrangian (2.10) and the strain energy (2.8) in combination with the special choices of material constants $(2.24)$ and (2.40). The antisymmetric part of the moment equilibrium ${ }^{6}$

$$
\partial^{i}\left(H_{[a j] i}-\lambda_{[a|i| j]}-\mu_{i[a j]}\right)=0,
$$

can be satisfied by the help of the choice of the coefficients

$$
d_{1}=0, \quad d_{2}-d_{3}=\frac{d_{0}}{2} .
$$

The physical interpretation of Eq. (2.42) is the following. Because we have required that the force stress should be symmetric, $\sigma_{[i j]}=0$, we have had to introduce the couple stresses $\lambda_{[a|i| j]}$ and $\mu_{i[a j]}$ in order to fulfill the antisymmetric moment equilibrium (2.42). Consequently, (2.42) expresses the moment equilibrium between the antisymmetric moment stresses $H_{[a j] i}, \lambda_{[a|i| j]}$ and $\mu_{i[a j]}$. The fact that the moment stress is the specific response to the presence of dislocations and that the field equation (2.38) expresses the moment equilibrium is a hint that the gauge theory of dislocations may be connected with the theory of a constrained, anholonomic Cosserat media with symmetric force stress. The dislocations bring the anholonomity into the

\footnotetext{
${ }^{6}$ Indices which are exempt from antisymmetrisation are enclosed by vertical bars.
} 
picture. A similar remark in this direction was given by Kluge [45]. It should be also possible to extend this theory to a more general dislocation theory in a general Cosserat continuum with antisymmetric force stress and 10 material constants which is not the aim of the present paper. However, for antisymmetric force stress and vanishing dislocation density the moment equilibrium equation recovers the form of the Cosserat equilibrium equation under zero external moments.

Finally, we may define the hyperstress

$$
M_{a}^{i j} \equiv-\frac{\partial \mathcal{L}}{\partial\left(\partial_{i} \beta^{a}{ }_{j}\right)}=H_{a}{ }^{i j}+\lambda_{a}{ }^{i j}+\mu_{a}^{i j} .
$$

This hyperstress tensor is close to Maugin's so-called "Piola-Kirchhoff" hyperstress [63]. Such a hyperstress tensor was also used by Toupin for the theory of nonsimple elastic materials [64]. Thus, the dislocation theory given in this paper can be considered as an anholonomic higher-order gradient theory in nonsimple materials. If $a_{1}=0$, there are no effects of hyperstresses and couple stresses.

By the help of the inverse of Hooke's law

$$
E_{i j}=\frac{1}{2 \mu}\left(\sigma_{i j}-\frac{\nu}{1+\nu} \delta_{i j} \sigma_{k}^{k}\right)
$$

and using the equilibrium condition $\partial^{j} \sigma_{i j}=0$, we obtain from Eq. (2.41) the inhomogeneous Helmholtz equation as field equation for every component of the stress tensor

$$
\left(1-\kappa^{-2} \Delta\right) \sigma_{i j}=\stackrel{\circ}{\sigma}_{i j}, \quad \kappa^{2}=\frac{2 \mu}{a_{1}} .
$$

It is important to note that (2.46) agrees with the field equation for the stress field in nonlocal elasticity $[28,30,31]$ and in gradient elasticity $[37,38]$. The factor $\kappa^{-1}$ has the physical dimension of a length and therefore it defines an internal characteristic length (dislocation length scale). By using (2.45) and (2.46) we obtain an inhomogeneous Helmholtz equation for the strain fields

$$
\left(1-\kappa^{-2} \Delta\right) E_{i j}=\stackrel{\circ}{E}_{i j}
$$

where $\stackrel{\circ}{E}_{i j}$ is the background strain tensor. Equation (2.47) is similar to the equation for strain of the gradient theory used by Gutkin and Aifantis [35-38], if we identify $\kappa^{-2}$ with their corresponding gradient coefficient (see, e.g., equation (4) in [37]). Note that only for compatible strain, $\phi_{i j}=0$, one can deduce an inhomogeneous Helmholtz equation for the displacement from (2.47) which is analogous to the equation for the displacement in gradient elasticity (see equation (3) in [37]).

The conditions on a gauge theoretical solution of dislocations in this framework are: (i) the stress field should have no singularity at $r=0$, (ii) the far field stress ought to be the classical stress field. We use the condition (ii) because the classical stress fields are well-established and in good agreement with physical observations, e.g. the investigation of stresses around dislocations by means of the photoelasticity method (see, e.g., [65-67]). 


\section{$3 \quad$ The gauge theoretical solution of the straight edge dislocation}

\subsection{The stress, strain and displacement field in an infinitely extended body}

Let us now derive the correct dislocation fields within the translational gauge theory. We take Cartesian coordinates, so that the $z$-axis is along the dislocation line and the $x$-axis is along the Burgers vector: $b^{x}=b, b^{y}=b^{z}=0$. The extra half plane lies in the plane $x=0$. In order to satisfy the equilibrium condition, we use the second order stress function $f$ and specialize to the plane problem of an edge dislocation by setting $\partial_{z} \equiv 0$. We are using the so-called stress function ansatz [26]

$$
\sigma_{i j}=\left(\begin{array}{ccc}
\partial_{y y}^{2} f & -\partial_{x y}^{2} f & 0 \\
-\partial_{x y}^{2} f & \partial_{x x}^{2} f & 0 \\
0 & 0 & p
\end{array}\right)
$$

with

$$
p=\nu \Delta f,
$$

where $\Delta$ denotes the two-dimensional Laplacian $\partial_{x x}^{2}+\partial_{y y}^{2}$. The relation (3.2) comes from the requirement: $E_{z z}=0$ (plane strain). Additionally, the strain is given in terms of the stress function as

$$
E_{i j}=\frac{1}{2 \mu}\left(\begin{array}{ccc}
\partial_{y y}^{2} f-\nu \Delta f & -\partial_{x y}^{2} f & 0 \\
-\partial_{x y}^{2} f & \partial_{x x}^{2} f-\nu \Delta f & 0 \\
0 & 0 & 0
\end{array}\right) .
$$

We use the classical stress field of a straight edge dislocation in terms of the Airy stress function as the background stress

$$
\stackrel{\circ}{\sigma}_{i j}=\left(\begin{array}{ccc}
\partial_{y y}^{2} \chi & -\partial_{x y}^{2} \chi & 0 \\
-\partial_{x y}^{2} \chi & \partial_{x x}^{2} \chi & 0 \\
0 & 0 & \nu \Delta \chi
\end{array}\right) .
$$

The Airy stress function [68-73]

$$
\chi=-A y \ln r, \quad A=\frac{\mu b}{2 \pi(1-\nu)},
$$

fulfills the following inhomogeneous bipotential (or biharmonic) equation

$$
\Delta \Delta \chi=-4 \pi A \partial_{y} \delta(r),
$$

where $\delta(r)$ is the two-dimensional Dirac delta function and $r^{2}=x^{2}+y^{2}$. Substituting (3.1) and (3.4) into (2.46) we get

$$
\left(\Delta-\kappa^{2}\right) f=\kappa^{2} A y \ln r .
$$

We make the ansatz

$$
f=-A y \ln r+f_{(1)}
$$

and obtain

$$
\left(\Delta-\kappa^{2}\right) f_{(1)}=2 A \partial_{y} \ln r
$$


Now we set

$$
f_{(1)}=2 A \partial_{y} f_{(2)}
$$

with

$$
\left(\Delta-\kappa^{2}\right) f_{(2)}=\ln r
$$

and we get the solution

$$
f_{(2)}=-\frac{1}{\kappa^{2}}\left(\ln r+K_{0}(\kappa r)\right) .
$$

Finally, we obtain the solution of (3.7)

$$
f=-A\left(y \ln r+\frac{2}{\kappa^{2}} \partial_{y}\left(\ln r+K_{0}(\kappa r)\right)\right) .
$$

Then the solution of the modified stress function of an edge dislocation with Burgers vector $b \| x$ reads $^{7}$

$$
f=-\frac{\mu b}{2 \pi(1-\nu)} y\left\{\ln r+\frac{2}{\kappa^{2} r^{2}}\left(1-\kappa r K_{1}(\kappa r)\right)\right\},
$$

where the first piece is the Airy stress function and $K_{n}$ is the modified Bessel function of the second kind and $n=0,1, \ldots$ denotes the order of this function.

By means of Eqs. (3.1) and (3.14), the modified stress of a straight edge dislocation is given by

$$
\begin{aligned}
\sigma_{x x}=-\frac{\mu b}{2 \pi(1-\nu)} \frac{y}{r^{4}}\left\{\left(y^{2}+3 x^{2}\right)\right. & +\frac{4}{\kappa^{2} r^{2}}\left(y^{2}-3 x^{2}\right) \\
& \left.-2 y^{2} \kappa r K_{1}(\kappa r)-2\left(y^{2}-3 x^{2}\right) K_{2}(\kappa r)\right\} \\
\sigma_{y y}=-\frac{\mu b}{2 \pi(1-\nu)} \frac{y}{r^{4}}\left\{\left(y^{2}-x^{2}\right)\right. & -\frac{4}{\kappa^{2} r^{2}}\left(y^{2}-3 x^{2}\right) \\
& \left.-2 x^{2} \kappa r K_{1}(\kappa r)+2\left(y^{2}-3 x^{2}\right) K_{2}(\kappa r)\right\}, \\
\sigma_{x y}=\frac{\mu b}{2 \pi(1-\nu)} \frac{x}{r^{4}}\left\{\left(x^{2}-y^{2}\right)-\right. & \frac{4}{\kappa^{2} r^{2}}\left(x^{2}-3 y^{2}\right) \\
& \left.-2 y^{2} \kappa r K_{1}(\kappa r)+2\left(x^{2}-3 y^{2}\right) K_{2}(\kappa r)\right\}, \\
\sigma_{z z}=-\frac{\mu b \nu}{\pi(1-\nu)} \frac{y}{r^{2}}\left\{1-\kappa r K_{1}(\kappa r)\right\} &
\end{aligned}
$$

The stress $\sigma_{z z}$ satisfies the condition $\sigma_{z z}=\nu\left(\sigma_{x x}+\sigma_{y y}\right)$. The trace of the stress tensor $\sigma_{k}^{k}$ produced by the edge dislocation in an isotropic medium is

$$
\sigma_{k}^{k}=-\frac{\mu b(1+\nu)}{\pi(1-\nu)} \frac{y}{r^{2}}\left\{1-\kappa r K_{1}(\kappa r)\right\} .
$$

The spatial distribution of stresses of an edge dislocation near the dislocation line

\footnotetext{
${ }^{7}$ For an edge dislocation with the Burgers vector $b \| y$ the modified stress function is given by:

$$
f=\frac{\mu b}{2 \pi(1-\nu)} x\left\{\ln r+\frac{2}{\kappa^{2} r^{2}}\left(1-\kappa r K_{1}(\kappa r)\right)\right\} .
$$
}




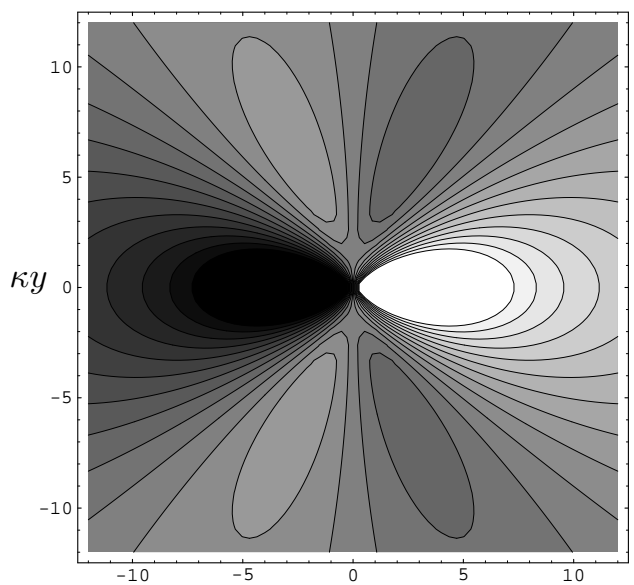

(a)
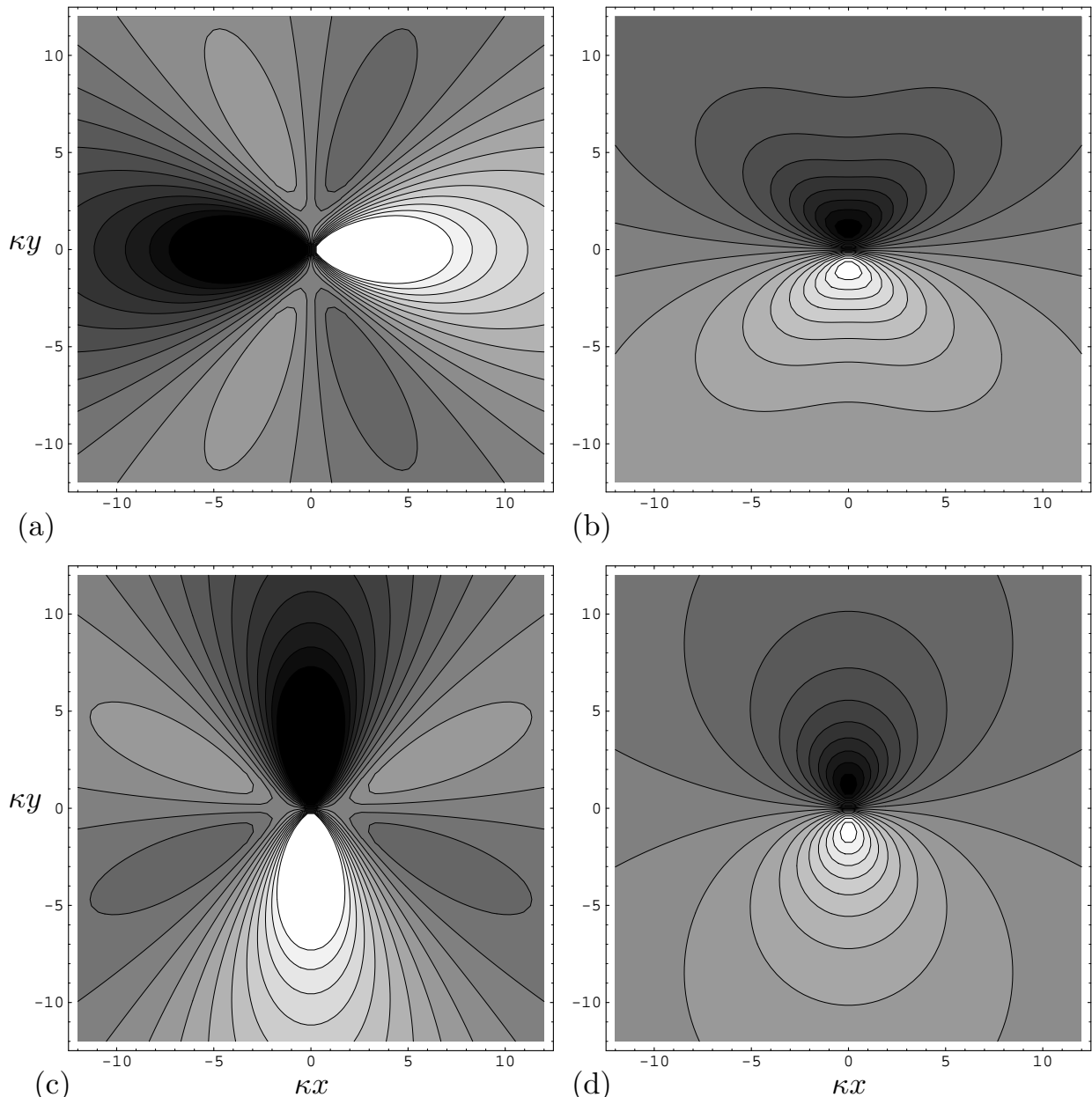

(b)

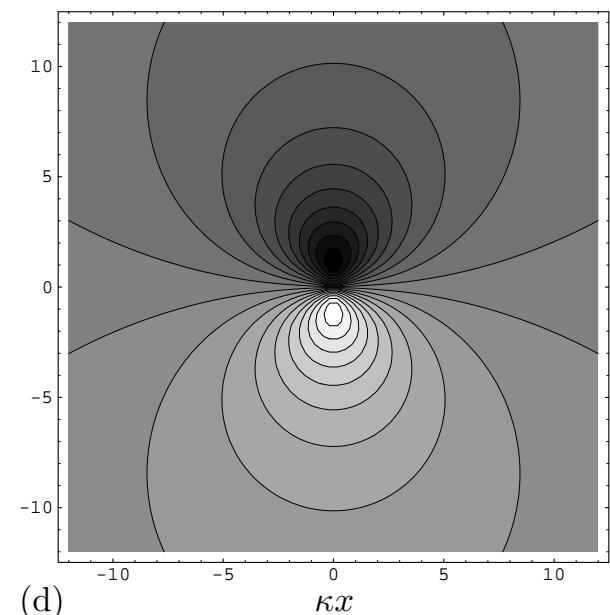

(d)

Figure 1: Stress contours of an edge dislocation near the dislocation line: (a) $\sigma_{x y}$, (b) $\sigma_{x x},(\mathrm{c}) \sigma_{y y},(\mathrm{~d}) \sigma_{z z}$.

are presented in Fig. 1.

Let us now discuss some details of the core modification of the stress fields. The stress fields have no artificial singularities at the core and the maximum stress occurs at a short distance away from the dislocation line (see Fig. 2). In fact, when $r \rightarrow 0$, we have

$$
K_{1}(\kappa r) \rightarrow \frac{1}{\kappa r}, \quad K_{2}(\kappa r) \rightarrow-\frac{1}{2}+\frac{2}{(\kappa r)^{2}},
$$

and thus $\sigma_{i j} \rightarrow 0$. It can be seen that the stresses have the following extreme values: $\left|\sigma_{x x}(0, y)\right| \simeq 0.546 \kappa \frac{\mu b}{2 \pi(1-\nu)}$ at $|y| \simeq 0.996 \kappa^{-1},\left|\sigma_{y y}(0, y)\right| \simeq 0.260 \kappa \frac{\mu b}{2 \pi(1-\nu)}$ at $|y| \simeq 1.494 \kappa^{-1},\left|\sigma_{x y}(x, 0)\right| \simeq 0.260 \kappa \frac{\mu b}{2 \pi(1-\nu)}$ at $|x| \simeq 1.494 \kappa^{-1}$, and $\left|\sigma_{z z}(0, y)\right| \simeq$ $0.399 \kappa \frac{\mu b \nu}{\pi(1-\nu)}$ at $|y| \simeq 1.114 \kappa^{-1}$. The stresses $\sigma_{x x}, \sigma_{y y}$ and $\sigma_{x y}$ are modified near the dislocation core $\left(0 \leq r \leq 12 \kappa^{-1}\right)$. Note that $\left|\sigma_{x y}(x, 0)\right| \simeq 0.260 \kappa \frac{\mu b}{2 \pi(1-\nu)}$ can be identified as the theoretical shear strength. The stress $\sigma_{z z}$ and the trace $\sigma_{k}^{k}$ are modified in the region: $0 \leq r \leq 6 \kappa^{-1}$. Far from the dislocation line $\left(r \gg 12 \kappa^{-1}\right)$ the gauge theoretical and the classical solutions of the stress of an edge dislocation coincide. Thus, the characteristic internal length $\kappa^{-1}$ determines the position and 

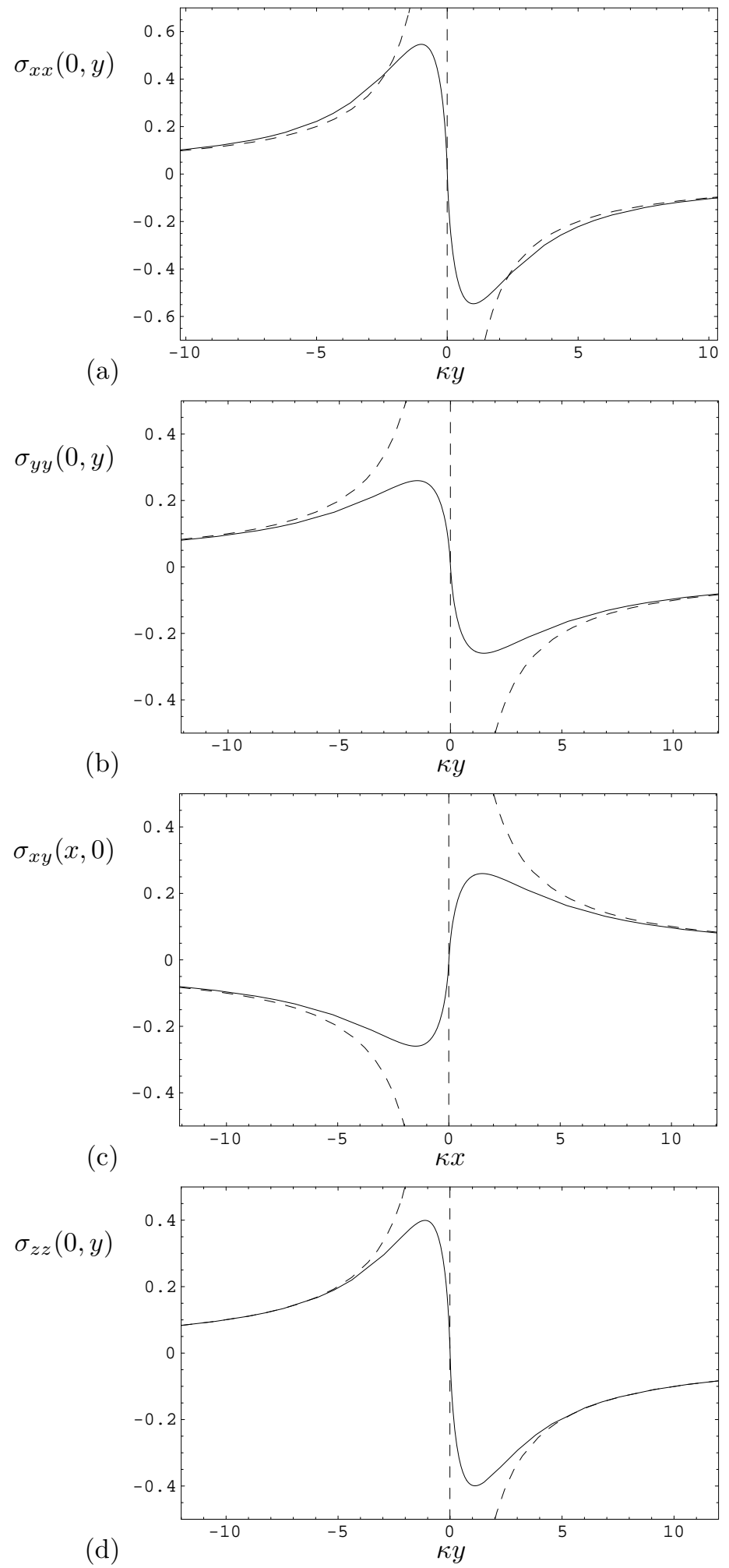

Figure 2: The stress components near the dislocation line: (a) $\sigma_{x x}(0, y),(\mathrm{b}) \sigma_{y y}(0, y)$, (c) $\sigma_{x y}(x, 0)$ are given in units of $\mu b \kappa /[2 \pi(1-\nu)]$ and (d) $\sigma_{z z}(0, y)$ is given in units of $\mu b \nu \kappa /[\pi(1-\nu)]$. The dashed curves represent the classical stress components. 
the magnitude of the stress maxima. It is interesting and important to note that the gauge-theoretical solutions (3.15)-(3.18) agree precisely with the gradient solutions given by Gutkin and Aifantis $[37,38]$ (with $\kappa^{-2}=c, c$ is the gradient coefficient).

For the elastic strain of an edge dislocation we find

$$
\begin{aligned}
E_{x x}=-\frac{b}{4 \pi(1-\nu)} \frac{y}{r^{2}}\{(1-2 \nu)+ & \frac{2 x^{2}}{r^{2}}+\frac{4}{\kappa^{2} r^{4}}\left(y^{2}-3 x^{2}\right) \\
& \left.-2\left(\frac{y^{2}}{r^{2}}-\nu\right) \kappa r K_{1}(\kappa r)-\frac{2}{r^{2}}\left(y^{2}-3 x^{2}\right) K_{2}(\kappa r)\right\}, \\
E_{y y}=-\frac{b}{4 \pi(1-\nu)} \frac{y}{r^{2}}\{(1-2 \nu)- & \frac{2 x^{2}}{r^{2}}-\frac{4}{\kappa^{2} r^{4}}\left(y^{2}-3 x^{2}\right) \\
E_{x y}=\frac{b}{4 \pi(1-\nu)} \frac{x}{r^{2}}\left\{1-\frac{2 y^{2}}{r^{2}}-\frac{4}{\kappa^{2} r^{4}}\left(x^{2}-3 y^{2}\right)\right. & \left.-\frac{2 y^{2}}{r^{2}} \kappa r K_{1}(\kappa r)+\frac{2}{r^{2}}\left(x^{2}-3 y^{2}\right) K_{2}(\kappa r)+\frac{2}{r^{2}}\left(y^{2}-3 x^{2}\right) K_{2}(\kappa r)\right\},
\end{aligned}
$$

which is in agreement with the solution given by Gutkin and Aifantis [36-38] in the framework of strain gradient elasticity. The plane-strain condition $E_{z z}=0$ of the classical dislocation theory is also valid in the dislocation core region. The components of the strain tensor have the following extreme values $(\nu=0.3):\left|E_{x x}(0, y)\right| \simeq$ $0.308 \kappa \frac{b}{4 \pi(1-\nu)}$ at $|y| \simeq 0.922 \kappa^{-1},\left|E_{y y}(0, y)\right| \simeq 0.010 \kappa \frac{b}{4 \pi(1-\nu)}$ at $|y| \simeq 0.218 \kappa^{-1}$, $\left|E_{y y}(0, y)\right| \simeq 0.054 \kappa \frac{b}{4 \pi(1-\nu)}$ at $|y| \simeq 4.130 \kappa^{-1}$, and $\left|E_{x y}(x, 0)\right| \simeq 0.260 \kappa \frac{b}{4 \pi(1-\nu)}$ at $|x| \simeq 1.494 \kappa^{-1}$. It is interesting to note that $E_{y y}(0, y)$ is much smaller than $E_{x x}(0, y)$ within the core region (see also $[37,38]$ ). The dilatation $E_{k}^{k}$ reads

$$
E_{k}^{k}=-\frac{b(1-2 \nu)}{2 \pi(1-\nu)} \frac{y}{r^{2}}\left\{1-\kappa r K_{1}(\kappa r)\right\} .
$$

It is convenient to rewrite the stress and strain fields in cylindrical coordinates. The stress function reads in cylindrical coordinates:

$$
f=-\frac{\mu b}{2 \pi(1-\nu)} \sin \varphi\left\{r \ln r+\frac{2}{\kappa^{2} r}\left(1-\kappa r K_{1}(\kappa r)\right)\right\}
$$

Here the stress function is related to the stresses by the equations

$$
\sigma_{r r}=\frac{1}{r} \partial_{r} f+\frac{1}{r^{2}} \partial_{\varphi \varphi}^{2} f, \quad \sigma_{\varphi \varphi}=\partial_{r r}^{2} f, \quad \sigma_{r \varphi}=-\partial_{r}\left(\frac{1}{r} \partial_{\varphi} f\right), \quad \sigma_{z z}=\nu\left(\sigma_{r r}+\sigma_{\varphi \varphi}\right) .
$$

In this way we find the stress of a straight edge dislocation in cylindrical coordinates

$$
\begin{aligned}
\sigma_{r r} & =-\frac{\mu b}{2 \pi(1-\nu)} \frac{\sin \varphi}{r}\left\{1-\frac{4}{\kappa^{2} r^{2}}+2 K_{2}(\kappa r)\right\}, \\
\sigma_{r \varphi} & =\frac{\mu b}{2 \pi(1-\nu)} \frac{\cos \varphi}{r}\left\{1-\frac{4}{\kappa^{2} r^{2}}+2 K_{2}(\kappa r)\right\}, \\
\sigma_{\varphi \varphi} & =-\frac{\mu b}{2 \pi(1-\nu)} \frac{\sin \varphi}{r}\left\{1+\frac{4}{\kappa^{2} r^{2}}-2 K_{2}(\kappa r)-2 \kappa r K_{1}(\kappa r)\right\}, \\
\sigma_{z z} & =-\frac{\mu b \nu}{\pi(1-\nu)} \frac{\sin \varphi}{r}\left\{1-\kappa r K_{1}(\kappa r)\right\},
\end{aligned}
$$


and the trace of the stress tensor reads

$$
\sigma_{k}^{k}=-\frac{\mu b(1+\nu)}{\pi(1-\nu)} \frac{\sin \varphi}{r}\left\{1-\kappa r K_{1}(\kappa r)\right\} .
$$

Eventually, the elastic strain of a straight edge dislocation is given in cylindrical coordinates as follows

$$
\begin{aligned}
& E_{r r}=-\frac{b}{4 \pi(1-\nu)} \frac{\sin \varphi}{r}\left\{(1-2 \nu)-\frac{4}{\kappa^{2} r^{2}}+2 K_{2}(\kappa r)+2 \nu \kappa r K_{1}(\kappa r)\right\} \\
& E_{r \varphi}=\frac{b}{4 \pi(1-\nu)} \frac{\cos \varphi}{r}\left\{1-\frac{4}{\kappa^{2} r^{2}}+2 K_{2}(\kappa r)\right\} \\
& E_{\varphi \varphi}=-\frac{b}{4 \pi(1-\nu)} \frac{\sin \varphi}{r}\left\{(1-2 \nu)+\frac{4}{\kappa^{2} r^{2}}-2 K_{2}(\kappa r)-2(1-\nu) \kappa r K_{1}(\kappa r)\right\} .
\end{aligned}
$$

The dilatation reads

$$
E_{k}^{k}=-\frac{b(1-2 \nu)}{2 \pi(1-\nu)} \frac{\sin \varphi}{r}\left\{1-\kappa r K_{1}(\kappa r)\right\} .
$$

Let us now calculate the distortion of an edge dislocation. The distortion $\beta^{a}{ }_{i}$ is given in terms of the stress function (3.14):

$$
\beta_{i}^{a}=\frac{1}{2 \mu}\left(\begin{array}{ccc}
\partial_{y y}^{2} f-\nu \Delta f & -\partial_{x y}^{2} f+2 \mu \omega & 0 \\
-\partial_{x y}^{2} f-2 \mu \omega & \partial_{x x}^{2} f-\nu \Delta f & 0 \\
0 & 0 & 0
\end{array}\right),
$$

where $\omega$ is used to express the antisymmetric part of the distortion, $\omega \equiv \beta_{[x y]}$. Eventually, $\omega$ is determined from the conditions:

$$
\begin{aligned}
& T_{x y}^{x}=\frac{1}{2 \mu}\left(2 \mu \partial_{x} \omega-(1-\nu) \partial_{y} \Delta f\right), \\
& T_{x y}^{y}=\frac{1}{2 \mu}\left(2 \mu \partial_{y} \omega+(1-\nu) \partial_{x} \Delta f\right) \equiv 0 .
\end{aligned}
$$

We find for the elastic distortion of the straight dislocation

$$
\begin{gathered}
\beta_{x}^{x}=-\frac{b}{4 \pi(1-\nu)} \frac{y}{r^{2}}\left\{(1-2 \nu)+\frac{2 x^{2}}{r^{2}}+\frac{4}{\kappa^{2} r^{4}}\left(y^{2}-3 x^{2}\right)\right. \\
\left.\quad-2\left(\frac{y^{2}}{r^{2}}-\nu\right) \kappa r K_{1}(\kappa r)-\frac{2}{r^{2}}\left(y^{2}-3 x^{2}\right) K_{2}(\kappa r)\right\} \\
\begin{array}{c}
\beta_{y}^{x}=\frac{b}{4 \pi(1-\nu)} \frac{x}{r^{2}}\left\{(3-2 \nu)-\frac{2 y^{2}}{r^{2}}-\frac{4}{\kappa^{2} r^{4}}\left(x^{2}-3 y^{2}\right)\right. \\
\left.\quad-2\left((1-\nu)+\frac{y^{2}}{r^{2}}\right) \kappa r K_{1}(\kappa r)+\frac{2}{r^{2}}\left(x^{2}-3 y^{2}\right) K_{2}(\kappa r)\right\}, \\
\beta_{x}^{y}=-\frac{b}{4 \pi(1-\nu)} \frac{x}{r^{2}}\left\{(1-2 \nu)+\frac{2 y^{2}}{r^{2}}+\frac{4}{\kappa^{2} r^{4}}\left(x^{2}-3 y^{2}\right)\right. \\
\left.\quad-2\left((1-\nu)-\frac{y^{2}}{r^{2}}\right) \kappa r K_{1}(\kappa r)-\frac{2}{r^{2}}\left(x^{2}-3 y^{2}\right) K_{2}(\kappa r)\right\}, \\
\beta_{y}^{y}=-\frac{b}{4 \pi(1-\nu)} \frac{y}{r^{2}}\left\{(1-2 \nu)-\frac{2 x^{2}}{r^{2}}-\frac{4}{\kappa^{2} r^{4}}\left(y^{2}-3 x^{2}\right)\right. \\
\left.\quad-2\left(\frac{x^{2}}{r^{2}}-\nu\right) \kappa r K_{1}(\kappa r)+\frac{2}{r^{2}}\left(y^{2}-3 x^{2}\right) K_{2}(\kappa r)\right\}
\end{array}
\end{gathered}
$$


and for the rotation

$$
\omega_{z} \equiv-\omega=-\frac{b}{2 \pi} \frac{x}{r^{2}}\left\{1-\kappa r K_{1}(\kappa r)\right\} .
$$

Eq. (3.43) is in agreement with the rotation vector calculated in the linear theory of dislocations in the Cosserat continuum [50]. The far fields of (3.39)-(3.43) are identical to the classical ones given in [74].

We now rewrite the distortions (3.39)-(3.42) as follows

$$
\begin{aligned}
\beta^{x} & =\frac{b}{2 \pi}\left\{\left(\left(1-\kappa r K_{1}(\kappa r)\right)+\frac{1}{2(1-\nu)} \cos 2 \varphi\left(1-\frac{4}{\kappa^{2} r^{2}}+2 K_{2}(\kappa r)\right)\right) \mathrm{d} \varphi\right. \\
& \left.+\frac{1}{2(1-\nu)} \sin 2 \varphi\left(\frac{4}{\kappa^{2} r^{3}}-\kappa K_{1}(\kappa r)-\frac{2}{r} K_{2}(\kappa r)\right) \mathrm{d} r\right\} \\
& \equiv \mathrm{d} u^{x}+\phi^{x} .
\end{aligned}
$$

We may interpret the field

$$
\phi^{x}=-\frac{b \kappa^{2}}{2 \pi} \varphi r K_{0}(\kappa r) \mathrm{d} r
$$

as the proper incompatible part (negative plastic distortion). This proper incompatible distortion is exactly the same as $\phi^{z}$ of a screw dislocation (see $\left.[2,3]\right)$. In components it reads

$$
\phi_{x}^{x}=-\frac{b \kappa^{2}}{2 \pi} x \varphi K_{0}(\kappa r), \quad \phi_{y}^{x}=-\frac{b \kappa^{2}}{2 \pi} y \varphi K_{0}(\kappa r) .
$$

The appearance of such proper incompatible distortion is a typical result of the gauge theory of dislocations. When $\kappa^{-1} \rightarrow 0$, the incompatible distortion (3.46) converts to $\phi_{x}^{x}=-b x \varphi \delta(r)$ and $\phi_{y}^{x}=-b y \varphi \delta(r)$. The compatible part of Eq. (3.44) is given in terms of the displacement field

$$
u^{x}=\frac{b}{2 \pi}\left\{\left(1-\kappa r K_{1}(\kappa r)\right) \varphi+\frac{1}{4(1-\nu)} \sin 2 \varphi\left(1-\frac{4}{\kappa^{2} r^{2}}+2 K_{2}(\kappa r)\right)\right\} .
$$

It is a modified displacement field (see Fig. 3a). We used $\varphi=\arctan (y / x)$ as a multivalued field. Note that the first part in (3.47) is different from the corresponding one given in gradient elasticity by Gutkin and Aifantis [36,37]. One reason is that they used a compatible elastic distortion. It is important to bear in mind that $u^{x}$ is not a simple arctan function as in the Volterra model of a dislocation. Additionally, we find from the distortion

$$
\begin{aligned}
& \beta^{y}= \frac{b}{4 \pi(1-\nu)}\left\{\sin 2 \varphi\left(1-\frac{4}{\kappa^{2} r^{2}}+2 K_{2}(\kappa r)\right) \mathrm{d} \varphi\right. \\
&\left.\quad-\left((1-2 \nu)\left(\frac{1}{r}-\kappa K_{1}(\kappa r)\right)+\cos 2 \varphi\left(\frac{4}{\kappa^{2} r^{3}}-\kappa K_{1}(\kappa r)-\frac{2}{r} K_{2}(\kappa r)\right)\right) \mathrm{d} r\right\} \\
& \equiv \mathrm{d} u^{y}+\phi^{y},
\end{aligned}
$$

the incompatible part

$$
\phi^{y}=0,
$$

and a single-valued displacement field

$$
u^{y}=-\frac{b}{8 \pi(1-\nu)}\left\{2(1-2 \nu)\left(\ln r+K_{0}(\kappa r)\right)+\cos 2 \varphi\left(1-\frac{4}{\kappa^{2} r^{2}}+2 K_{2}(\kappa r)\right)\right\}
$$



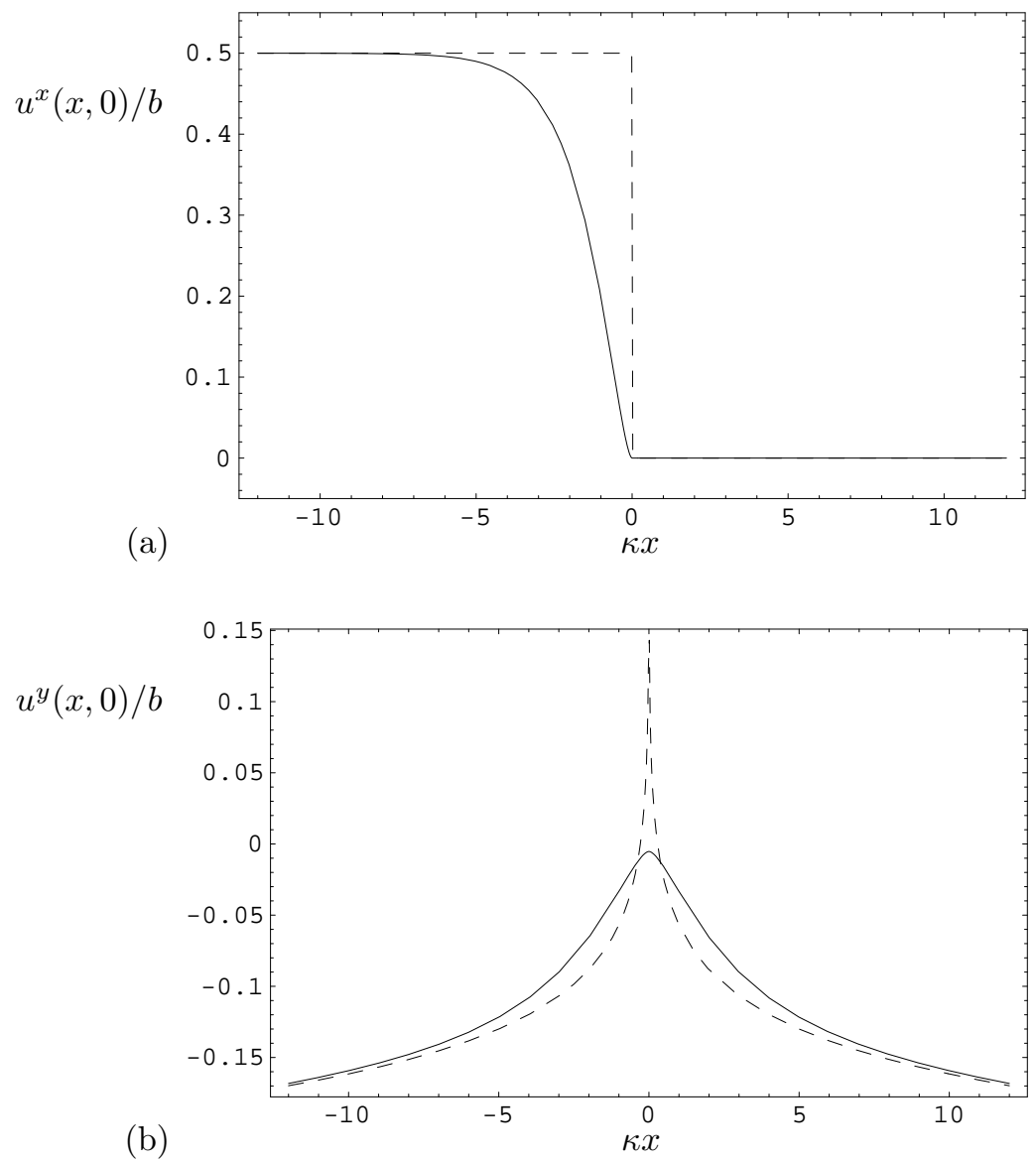

Figure 3: Effective displacement fields $(\nu=0.3): u^{x}(x, y \rightarrow+0) / b,(\mathrm{~b}) u^{y}(x, 0) / b$. The dashed curves represent the classical solution.

which has no singularity (see Fig. 3b). This means that $\beta^{y}$ is a proper compatible distortion. That is the reason why the field $u^{y}$ agrees precisely with the corresponding formula in gradient elasticity (see [36,37]). The displacement field $u^{y}$ differs from the classical one in the region $0 \leq r \leq 12 \kappa^{-1}$. In this framework the displacement fields (3.47) and (3.50) have no singularity. The far fields of the displacements (3.47) and (3.50) are identical to the classical ones given in $[26,75,76]$. It can be seen that the solution (3.47) leads to an asymmetric smoothing of the displacement profile (see Fig. 3a). In fact, it is asymmetric around the dislocation line. Note that the incompatible distortion (3.46) also has an asymmetric form. These asymmetries would lead to an unexpected asymmetry of the dislocation core. From the mathematical point of view, the asymmetry is induced by the decomposition of the elastic distortion (2.2) into compatible and incompatible parts. Keep in mind that such a decomposition is gauge invariant (see Eq. (2.3)) and we have a local translation as an additional degree of freedom. This gauge invariance can be used to improve the situation. So far we have considered $\varphi$ as a multi-valued field. On the other hand, in the defect theory $[35-38,74] \varphi$ is usually used as single-valued and discontinuous function. It is made unique by cutting the half-plane $y=0$ at $x<0$ and assuming $\varphi$ to jump from $\pi$ to $-\pi$ when crossing the cut. If we use the single-valued discontinuous form for $\varphi$ 


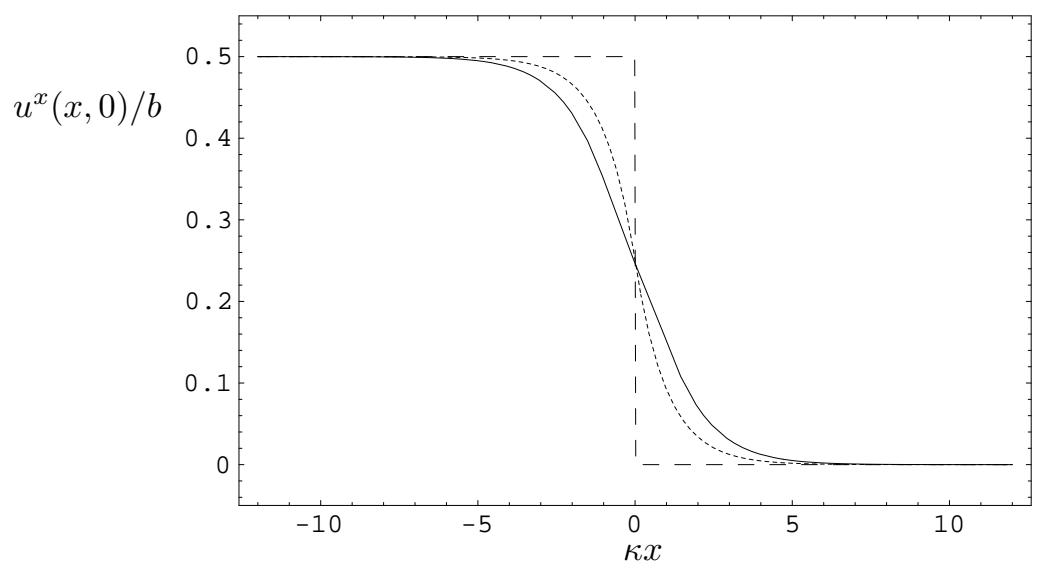

Figure 4: Displacement $u^{x}(x, y \rightarrow+0) / b$ near the line of edge dislocation within gauge model (solid), the gradient model (small dashed) and the classical elasticity (dashed). We used $\kappa^{2}=1 / c$.

and the local gauge transformation (2.3) with the gauge function

$$
\tau^{x}=\frac{b}{2 \pi} \operatorname{sign}(y) \frac{\pi}{2} \kappa r K_{1}(\kappa r)
$$

we obtain for the improved displacement field

$$
\begin{aligned}
u^{x}=\frac{b}{2 \pi}\{\varphi(1- & \left.\kappa r K_{1}(\kappa r)\right)+\frac{\pi}{2} \operatorname{sign}(y) \kappa r K_{1}(\kappa r) \\
& \left.+\frac{1}{2(1-\nu)} \frac{x y}{r^{2}}\left(1-\frac{4}{\kappa^{2} r^{2}}+2 K_{2}(\kappa r)\right)\right\},
\end{aligned}
$$

and for the proper incompatible distortion

$$
\begin{aligned}
\phi_{x}^{x} & =-\frac{b}{2 \pi} \kappa^{2} x K_{0}(\kappa r)\left(\varphi-\frac{\pi}{2} \operatorname{sign}(y)\right) \\
\phi_{y}^{x} & =-\frac{b}{2 \pi}\left\{\kappa^{2} y K_{0}(\kappa r)\left(\varphi-\frac{\pi}{2} \operatorname{sign}(y)\right)+\pi \delta(y)\left(1-\operatorname{sign}(x)\left[1-\kappa r K_{1}(\kappa r)\right]\right)\right\}
\end{aligned}
$$

Now the displacements and the incompatible distortions are symmetric in the core region around the dislocation line ${ }^{8}$. It is interesting to compare the displacement field (3.52) with the displacement calculated by Gutkin and Aifantis. When $y \rightarrow 0$, the

\footnotetext{
${ }^{8}$ Note that in the case of a screw dislocation the symmetrical displacement and the corresponding incompatible distortion read, respectively,

$$
u^{z}=\frac{b}{2 \pi}\left\{\varphi\left(1-\kappa r K_{1}(\kappa r)\right)+\frac{\pi}{2} \operatorname{sign}(y) \kappa r K_{1}(\kappa r)\right\},
$$

and

$$
\phi_{x}^{z}=-\frac{b}{2 \pi} \kappa^{2} x K_{0}(\kappa r)\left(\varphi-\frac{\pi}{2} \operatorname{sign}(y)\right)
$$$$
\phi_{y}^{z}=-\frac{b}{2 \pi}\left\{\kappa^{2} y K_{0}(\kappa r)\left(\varphi-\frac{\pi}{2} \operatorname{sign}(y)\right)+\pi \delta(y)\left(1-\operatorname{sign}(x)\left[1-\kappa r K_{1}(\kappa r)\right]\right)\right\} .
$$ 


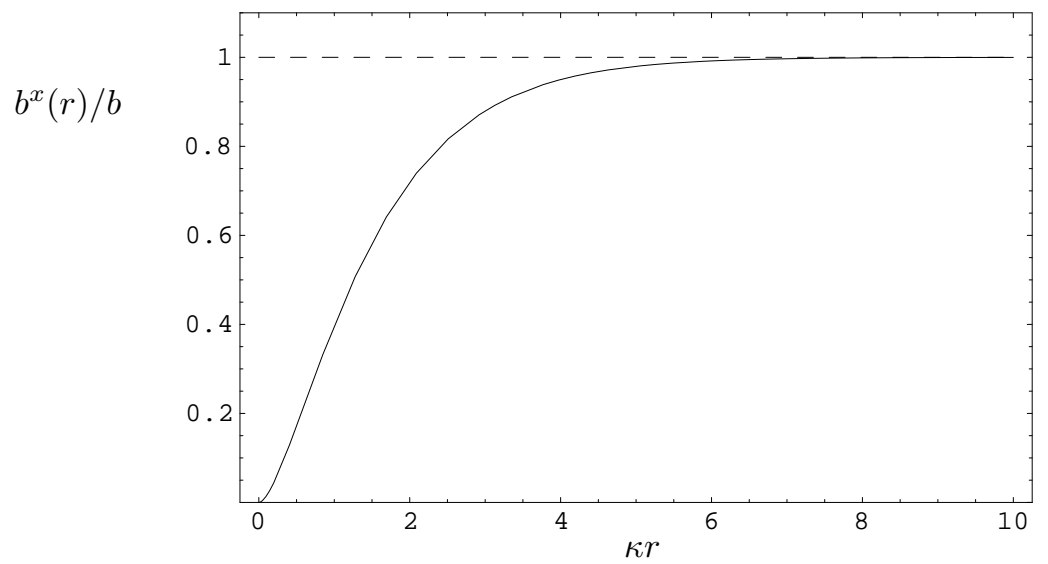

Figure 5: Effective Burgers vector $b^{x}(r) / b$ (solid).

Bessel function terms in (3.52) lead to the symmetric smoothing of the displacement profile in contrast to the abrupt jump occuring in the profile of the classical solution (see Fig. 4). When $y \rightarrow 0$, the displacement $u^{x}(x, y)$ calculated by Gutkin and Aifantis $[36,38]$ within the gradient model has the explicit form

$$
u^{x}(x, y \rightarrow 0)=\frac{b}{2 \pi} \operatorname{sign}(y) \frac{\pi}{2}\left\{1-\operatorname{sign}(x)\left(1-\mathrm{e}^{-|x| / \sqrt{c}}\right)\right\} .
$$

The exponential term which appears in the gradient solution (3.55) leads to the smoothing of the displacement profile (see Fig. 4). The size of the transition zone is approximately $12 \kappa^{-1}$ which gives the value $r_{c} \simeq 6 \kappa^{-1}$ for the core radius. Consequently, in the gauge theory of dislocations and in gradient elasticity the dislocation core appears naturally. Therefore, these displacement fields may be used to model the dislocation core. In this way, one can compare the calculated dislocation core with HRTEM micrographs and related computer simulations of the core region. It would be very interesting to check a possible material dependence of $\kappa^{-1}$.

Finally, the effective Burgers vector can be calculated as

$$
b^{x}(r)=\oint_{\gamma} \beta^{x}=b\left\{1-\kappa r K_{1}(\kappa r)\right\} .
$$

This effective Burgers vector $b^{x}(r)$ differs from the constant Burgers vector $b$ in the region $0 \leq r \leq 6 \kappa^{-1}$ (see Fig. 5). For the value of $\kappa^{-1}=0.25 a$ the core radius is $r_{c}=1.5 a$ and for the value of $\kappa^{-1}=0.399 a$ the core radius is $r_{c}=2.4 a$. Note that the effective Burgers vector $b^{x}(r)$ of an edge dislocation has the same form as the effective Burgers vector $b^{z}(r)$ of a screw dislocation which is obtained in $[2,4,9,10]$.

\subsection{The dislocation density, moment stress and core energy}

The proper incompatible part of the elastic distortion gives rise to a localized torsion and moment stress tensor. We find for the torsion

$$
T_{x y}^{x}=-T_{y x}^{x}=\frac{b \kappa^{2}}{2 \pi} K_{0}(\kappa r)
$$

and the dislocation density of an edge dislocation

$$
\alpha^{x z}=\frac{b \kappa^{2}}{2 \pi} K_{0}(\kappa r)
$$


which satisfies the translational Bianchi identity: $\partial_{i} \alpha^{a i}=0$. On can see that the dislocation density is short-reaching. It is interesting to note that the torsion $T_{x y}^{x}$ of an edge dislocation has the same form as the torsion $T_{x y}^{z}$ of a screw dislocation which is given in $[2-4,9,10]$. In the limit as $\kappa^{-1} \rightarrow 0$, the gauge theoretical result (3.58) converts to the classical dislocation density $\alpha^{x z}=b \delta(r)$. Additionally, the dislocation density (3.58) agrees with Eringen's two-dimensional nonlocal modulus (nonlocal kernel) used in [28-31]. Consequently, the dislocation density tensor is Green's function of the Helmholtz equation:

$$
\left(1-\kappa^{-2} \Delta\right) \alpha^{x z}(r)=b \delta(r) .
$$

This torsion gives rise to a localized moment stress. The localized moment stress of bending type is given by the help of (2.27) as

$$
H_{x z}=\frac{\mu b}{\pi(1-\nu)} K_{0}(\kappa r), \quad H_{z x}=-\frac{\mu b \nu}{\pi(1-\nu)} K_{0}(\kappa r) .
$$

This expression is very close to the moment stress of an edge dislocation given in [77]. By means of the dislocation density and the moments stress we are able to calculate the core energy of an edge dislocation. The dislocation core energy density is given by

$$
V_{\text {core }}=\frac{1}{2} \alpha^{a i} H_{a i}=\frac{\mu b^{2} \kappa^{2}}{4 \pi^{2}(1-\nu)} K_{0}(\kappa r)^{2} .
$$

Obviously, the moment stress $H_{z x}$ gives no contribution to the dislocation core energy. We obtain for the dislocation core energy per unit length of the straight edge dislocation

$$
\begin{aligned}
E_{\text {core }} & =\int_{0}^{2 \pi} \mathrm{d} \varphi \int_{0}^{R} r V_{\text {core }} \mathrm{d} r \\
& =\left.\frac{\mu b^{2} \kappa^{2}}{4 \pi(1-\nu)} r^{2}\left\{K_{0}(\kappa r)^{2}-K_{1}(\kappa r)^{2}\right\}\right|_{0} ^{R} \\
& =\frac{\mu b^{2}}{4 \pi(1-\nu)}\left\{1+\kappa^{2} R^{2}\left(K_{0}(\kappa R)^{2}-K_{1}(\kappa R)^{2}\right)\right\},
\end{aligned}
$$

where $R$ denotes the size of the solid body. In the limit $R \rightarrow \infty$, the core energy of the straight edge dislocation,

$$
E_{\text {core }}=\frac{\mu b^{2}}{4 \pi(1-\nu)},
$$

coincides with the core energy that is calculated for the edge dislocation in the PeierlsNabarro model [26]. The core energy is different from the constant value in the region $0 \leq \kappa R \leq 3$.

The far-reaching rotation gradients read

$$
\begin{aligned}
& k_{x y}^{x}=-k_{y x}^{x}=-\frac{b}{2 \pi r^{4}}\left\{\left(x^{2}-y^{2}\right)\left(1-\kappa r K_{1}(\kappa r)\right)-\kappa^{2} x^{2} r^{2} K_{0}(\kappa r)\right\}, \\
& k_{x y}^{y}=-k_{y x}^{y}=-\frac{b}{2 \pi r^{4}} x y\left\{2\left(1-\kappa r K_{1}(\kappa r)\right)-\kappa^{2} r^{2} K_{0}(\kappa r)\right\} .
\end{aligned}
$$

The form of these rotation gradients is in agreement with the expressions calculated within the theory of Cosserat media (see $[48,51]$ ). 


\subsection{The straight edge dislocation in a cylinder}

Now we consider a straight edge dislocation in a cylinder of finite outer radius $R$ with an outer boundary free from external forces. An advantage in our framework is that we do not need an inner radius because the stress and strain fields are zero at $r=0$ in quite natural way. If the outer boundary is to be free, it is necessary to add to the stress function (3.5) an additional stress function $\chi^{\prime}=A C y r^{2}$. Then the modified stress function is of the form

$$
f=-\frac{\mu b}{2 \pi(1-\nu)} \sin \varphi\left\{r \ln r+\frac{2}{\kappa^{2} r}\left(1-\kappa r K_{1}(\kappa r)-4 C r^{2}\right)-C r^{3}\right\},
$$

where the constant $C$ is determined by the semi-classical boundary condition that the stresses $\sigma_{r r}$ and $\sigma_{r \varphi}$ should be zero at $r=R$. In this way we find the stress of the edge dislocation in cylindrical coordinates

$$
\begin{aligned}
\sigma_{r r} & =-\frac{\mu b}{2 \pi(1-\nu)} \frac{\sin \varphi}{r}\left\{1-\frac{4}{\kappa^{2} r^{2}}+2 K_{2}(\kappa r)-2 C r^{2}\right\}, \\
\sigma_{r \varphi} & =\frac{\mu b}{2 \pi(1-\nu)} \frac{\cos \varphi}{r}\left\{1-\frac{4}{\kappa^{2} r^{2}}+2 K_{2}(\kappa r)-2 C r^{2}\right\}, \\
\sigma_{\varphi \varphi} & =-\frac{\mu b}{2 \pi(1-\nu)} \frac{\sin \varphi}{r}\left\{1+\frac{4}{\kappa^{2} r^{2}}-2 K_{2}(\kappa r)-2 \kappa r K_{1}(\kappa r)-6 C r^{2}\right\}, \\
\sigma_{z z} & =-\frac{\mu b \nu}{\pi(1-\nu)} \frac{\sin \varphi}{r}\left\{1-\kappa r K_{1}(\kappa r)-4 C r^{2}\right\},
\end{aligned}
$$

with

$$
C=\frac{1}{2 R^{2}}\left\{1-\frac{4}{\kappa^{2} R^{2}}+2 K_{2}(\kappa R)\right\} .
$$

The corresponding trace is given by

$$
\sigma_{k}^{k}=-\frac{\mu b(1+\nu)}{\pi(1-\nu)} \frac{\sin \varphi}{r}\left\{1-\kappa r K_{1}(\kappa r)-4 C r^{2}\right\} .
$$

The elastic strain of this edge dislocation in a cylinder is

$$
\begin{aligned}
& E_{r r}=-\frac{b}{4 \pi(1-\nu)} \frac{\sin \varphi}{r}\left\{(1-2 \nu)-\frac{4}{\kappa^{2} r^{2}}+2 K_{2}(\kappa r)+2 \nu \kappa r K_{1}(\kappa r)-(2-8 \nu) C r^{2}\right\}, \\
& E_{r \varphi}=\frac{b}{4 \pi(1-\nu)} \frac{\cos \varphi}{r}\left\{1-\frac{4}{\kappa^{2} r^{2}}+2 K_{2}(\kappa r)-2 C r^{2}\right\} \\
& E_{\varphi \varphi}=-\frac{b}{4 \pi(1-\nu)} \frac{\sin \varphi}{r}\left\{(1-2 \nu)+\frac{4}{\kappa^{2} r^{2}}-2 K_{2}(\kappa r)-2(1-\nu) \kappa r K_{1}(\kappa r)\right. \\
& \left.\quad-(6-8 \nu) C r^{2}\right\}
\end{aligned}
$$

The corresponding dilatation field reads

$$
E_{k}^{k}=-\frac{b(1-2 \nu)}{2 \pi(1-\nu)} \frac{\sin \varphi}{r}\left\{1-\kappa r K_{1}(\kappa r)-4 C r^{2}\right\} .
$$

From the condition (3.38) we find for the rotation of an edge dislocation in a cylinder

$$
\omega_{z} \equiv-\omega=-\frac{b}{2 \pi} \frac{\cos \varphi}{r}\left\{1-\kappa r K_{1}(\kappa r)+4 C r^{2}\right\}
$$


which gives an additional constant contribution to the bend-twist tensor $k_{x y}^{x}$ in Eq. (3.64). The dislocation density of the edge dislocation in a cylinder is determined by the condition (3.37) and it has the same form as the dislocation in an infinitely extended body (3.58).

For convenience we give the displacement components in Cartesian coordinates

$$
\begin{aligned}
& u^{x}=\frac{b}{2 \pi}\left\{\varphi\left(1-\kappa r K_{1}(\kappa r)\right)+\frac{\pi}{2} \operatorname{sign}(y) \kappa r K_{1}(\kappa r)\right. \\
& \left.\quad+\frac{1}{2(1-\nu)} \frac{x y}{r^{2}}\left(1-\frac{4}{\kappa^{2} r^{2}}+2 K_{2}(\kappa r)\right)+\frac{C(3-4 \nu)}{(1-\nu)} x y\right\}, \\
& u^{y}=-\frac{b}{4 \pi(1-\nu)}\left\{(1-2 \nu)\left(\ln r+K_{0}(\kappa r)\right)+\frac{x^{2}-y^{2}}{2 r^{2}}\left(1-\frac{4}{\kappa^{2} r^{2}}+2 K_{2}(\kappa r)\right)\right. \\
& \left.\quad+C\left(x^{2}(5-4 \nu)-y^{2}(1-4 \nu)\right)\right\} .
\end{aligned}
$$

Note that this boundary-value problem of the edge dislocation may be applied to describe dislocation behaviour in nanoparticles and nanowires.

\section{The relation to the nonlocal theory of dislocations}

Let me now give the relation to Eringen's so-called nonlocal theory of dislocations [28, $30,31]$. It includes the effect of long range interatomic forces so that it can be used as a continuum model of the atomic lattice dynamics. Using Green's function of the Helmholtz equation (3.59) we may solve the field equation for every component of the stress field (2.46) by the help of the convolution integral:

$$
\sigma_{i j}(r)=\int_{V} G\left(r-r^{\prime}\right) \stackrel{\circ}{\sigma}_{i j}\left(r^{\prime}\right) \mathrm{d} v\left(r^{\prime}\right)
$$

with

$$
G\left(r-r^{\prime}\right) \equiv \frac{1}{b} \alpha^{x z}\left(r-r^{\prime}\right)=\frac{\kappa^{2}}{2 \pi} K_{0}\left(\kappa\left(r-r^{\prime}\right)\right) .
$$

In this way we deduce Eringen's so-called nonlocal constitutive relation for a linear homogeneous, isotropic solid. Therefore, the gauge theoretical description of dislocations in this paper is close to the nonlocal elasticity of dislocations $[28,30,31]$, if one uses the special two-dimensional Green's function (4.2). The form of the nonlocal kernel (dislocation density) is fixed by the incompatible distortion. Finally, we may conclude that the gauge theory of dislocation contains a weak nonlocality due to the moment stresses in the dislocation core region. This observation is in agreement with Kröner's opinion that the introduction of moment stresses represents the first step in a transition from local to a nonlocal elasticity theory [77-80].

The characteristic internal length in these theories is $\kappa^{-1}$. This length may be selected to be proportional to the lattice parameter $a$ for a single crystal, i.e.

$$
\kappa^{-1}=e_{0} a,
$$

where $e_{0}$ is a non-dimensional constant or material function which can be determined by one experiment [30]. For $e_{0}=0$ we have classical elasticity and there are no effects of moment stresses. In this limit the nonlocal kernel must revert to the Dirac delta measure. In $[28,30]$ the choice $e_{0}=0.399$ and in $[32-34]$ the choice $e_{0}=0.25$ are proposed (see also $[2,3,38]$ ). 
Consequently, we can conclude that the gauge theoretical solution of the stress field for an edge dislocation given in this paper is also a solution in nonlocal elasticity. However, a difference to our approach is that Eringen's nonlocal theory works with compatible strain instead of incompatible strain and the strains and displacements have the classical form with singularities at the dislocation line.

\section{Discussion and Conclusion}

The translational gauge theory of dislocations has been employed to consider the straight edge dislocation in an infinitely extended body and in a cylinder. This translational gauge theory unifies elasticity and plasticity to elastoplasticity in field theoretical way. In this framework, elastoplasticity is a theory with force stresses and moment stresses. The size of this moment stress can be calculated even from linearized elastoplasticity theory. Using the stress function method, exact analytical solutions for the displacements, strain and stress fields have been derived which have not any artificial divergency at the dislocation core and the corresponding far fields agree with the classical ones for the edge dislocation. The strain and stress fields of an edge dislocation in an infinitely extended body are in exact agreement with those obtained by Gutkin and Aifantis [36,37] through an analysis of the edge dislocation in the theory of gradient elasticity. Nevertheless, the gauge theoretical and the gradient theoretical approaches are different and they give slightly different results, e.g. the displacement field $u^{x}$ due to the incompatible distortion in the gauge theory of dislocations. On the other hand, the strain gradient elasticity used a purely compatible distortion. However, the difference cannot be too big and the numerical values should be of the same order. The dislocation density of an edge dislocation obtained in the gauge theoretical framework is in agreement with the nonlocal kernel given by Ari and Eringen [28-31]. From that point of view the nonlocal elasticity theory approach is close to ours. But, on the other hand, Eringen's nonlocal elasticity contains compatible strains only and the strain and displacement fields have the same form as in a classical elasticity. In the gauge theoretical analysis the dislocation core arises naturally. We have calculated the localized moment stress which is the response to dislocation density and the far-reaching rotation gradient (bend-twist tensor).

The dislocation model used in this paper contains, in general, 10 material constants. The total Lagrangian of this model is given in terms of the distortion, the dislocation density (torsion tensor) and deWit's bend-twist tensor. By the help of some special choices $(2.24),(2.40)$ and (2.43) we reduced the number of constants to 3 material constants, namely 2 elastic constants and 1 internal length. As a result we obtain a dislocation theory with symmetric force stresses. The special choice (2.24) for the three coefficients $a_{1}, a_{2}$ and $a_{3}$ in the dislocation gauge Lagrangian gives the core energy of the edge dislocation in an infinite medium which is in agreement with the expression obtained in the Peierls-Nabarro model. It is important to note that one can also use the total Lagrangian of this dislocation model for the gauge theoretical description of screw dislocations. In this turn, one reproduces all quantities of the screw dislocation calculated in [2]. Therefore, by using the Lagrangian proposed in this paper, one is able to obtain gauge theoretical solutions for the edge and screw dislocation which have no singularities and agree with the classical solutions in the far field. The (linear) gauge equation formulated in terms of the stress field is an inhomogeneous Helmholtz equation for the screw and the edge dislocation.

It will be interesting to apply the nonsingular solutions of the edge and screw dislocation to dislocation modelling. One can use the gauge theoretical displacement fields to simulate the core region for HRTEM micrographs. Another interesting ap- 
plication could be the x-ray diffraction profile analysis. One should use the materials tungsten, diamond or aluminium because they are nearly isotropic. In this way the coefficient $\kappa^{-1}$ should be experimentally verified. Additionally, it would be of interest to compare experimental results with the values from different theoretical approaches (gauge theory, strain gradient elasticity and/or nonlocal elasticity). Another possibility to determine the coefficient $\kappa^{-1}$ should be a lattice-theoretical approach. The coefficient $\kappa^{-1}$ expresses the interactions and the properties in the core which are different from those in the undeformed crystal. Consequently, the elastic moduli say nothing about the interactions in the core of dislocations. It should be possible to

calculate $\kappa^{-1}$ from the coupling parameters of the lattice at the dislocation core.

\section{Acknowledgement}

The author wishes to thank Professors Friedrich W. Hehl, Stefan Müller and Alfred Seeger and Dr. Gerald Wagner for stimulating discussions, furthermore to Drs. Mikhail Yu. Gutkin and Cyril Malyshev for correspondence and useful remarks. He would like to express his gratitude to the Max-Planck-Institut für Mathematik in den Naturwissenschaften for the fine conditions of work and for financial support.

\section{References}

[1] M. Lazar, Ann. Phys. (Leipzig) 9 (2000) 461.

[2] M. Lazar, J. Phys. A: Math. Gen. 35 (2002) 1983.

[3] M. Lazar, Ann. Phys. (Leipzig) 11 (2002) 635.

[4] C. Malyshev, Ann. Phys. (N.Y.) 286 (2000) 249.

[5] A.A. Golebiewska-Lasota, Int. J. Engng. Sci. 17 (1979) 329.

[6] D.G.B. Edelen, Int. J. Engng. Sci. 20 (1982) 49.

[7] A. Kadić and D.G.B. Edelen, A Gauge Theory of Dislocations and Disclinations, in: Lecture Notes in Physics, Vol. 174, Springer, Berlin (1983).

[8] D.G.B. Edelen and D.C. Lagoudas, Gauge Theory and Defects in Solids, NorthHolland, Amsterdam (1988).

[9] M.C. Valsakumar and D. Sahoo, Bull. Mater. Sci. 10 (1988) 3.

[10] D.G.B. Edelen, Int. J. Engng. Sci. 34 (1996) 81.

[11] V.L. Popov, Int. J. Engng. Sci. 30 (1992) 329.

[12] A. Kadić-Galeb and R.C. Batra, Int. J. Engng. Sci. 32 (1994) 291.

[13] M.O. Katanaev and I.V. Volovich, Ann. Phys. (N.Y.) 216 (1992) 1.

[14] B.K.D. Gairola, Gauge Invariant Formulation of Continuum Theory of Defects, in: Continuum Models and Discrete Systems, Proc. 4th Int. Symp., O. Brulin and R.K.T. Hsieh, eds., North-Holland, Amsterdam (1981) p. 55.

[15] B.K.D. Gairola, Gauge Theory of Dislocations, in: Continuum Models and Discrete Systems, Proc. 7th Int. Symp., Paderborn, Germany, K.-H. Anthony and H.-J. Wagner, eds., Trans. Techn. Publ., Aedermannsdorf (CH) (1993) p. 579. 
[16] E. Kröner, A Variational Principle in Nonlinear Dislocations Theory, in: Proc. 2nd Int. Conf. Nonlin. Mechanics, Chien Wei-zang, ed., Peking University Press, Beijing (1993) p. 59.

[17] E. Kröner, Dislocation Theory as a Physical Field Theory, in: Continuum Models and Discrete Systems, Proc. 8th Int. Symp., Varna, Bulgaria, K.Z. Markov, ed., World Scientific, Singapore (1996) p. 522.

[18] I.A. Kunin, On the Gauge Theory of Dislocations, in: The Mechanics of Dislocations, Eds. E.C. Aifantis and J.P. Hirth, American Society of Metals, Metals Park, Ohio (1985) p. 69.

[19] H. Kleinert, Gauge Fields in Condensed Matter Vol. II: Stresses and Defects, World Scientific, Singapore (1989).

[20] V.A. Osipov, J. Phys. A: Math. Gen. 24 (1991) 3237.

[21] N.B. Volkov, J. Phys. A: Math. Gen. 30 (1997) 6391.

[22] N.M. Bogatov, Phys. Stat. Sol. (b) 228 (2001) 651.

[23] R.E. Peierls, Proc. Phys. Soc. (London) 54 (1940) 34.

[24] F.R.N. Nabarro, Proc. Phys. Soc. (London) 59 (1947) 256.

[25] F.R.N. Nabarro, Theory of Crystal Dislocations, Dover, New York (1987).

[26] J.P. Hirth and J. Lothe, Theory of Dislocations, John Wiley, New York (1982).

[27] A.C. Eringen, Int. J. Engng. Sci. 15 (1977) 177.

[28] A.C. Eringen, J. Appl. Phys. 54 (1983) 4703.

[29] N. Ari and A.C. Eringen, Cryst. Lattice Defects Amorph. Mat. 10 (1983) 33.

[30] A.C. Eringen, Nonlocal Continuum Theory for Dislocations and Fracture, in: The Mechanics of Dislocations, Eds. E.C. Aifantis and J.P. Hirth, American Society of Metals, Metals Park, Ohio (1985) p. 101.

[31] A.C. Eringen, Res. Mech. 21 (1987) 313.

[32] B.S. Altan and E.C. Aifantis, Scripta Metall. Mater. 26 (1992) 319.

[33] E.C. Aifantis, J. Mechan. Behav. Mater. 5 (1994) 355.

[34] B.S. Altan and E.C. Aifantis, J. Mech. Behav. Mater. 8 (1997) 231.

[35] M.Yu. Gutkin and E.C. Aifantis, Scripta Mater. 35 (1996) 1353.

[36] M.Yu. Gutkin and E.C. Aifantis, Scripta Mater. 36 (1997) 129.

[37] M.Yu. Gutkin and E.C. Aifantis, Scripta Mater. 40 (1999) 559.

[38] M.Yu. Gutkin, Rev. Adv. Mater. Sci. 1 (2000) 27.

[39] A.D. Brailsford, Phys. Rev. 142 (1966) 383.

[40] I.A. Kunin, Theory of Elastic Media with Microstructure, Springer, Berlin (1986).

[41] G. Vörös and I. Kovács, Phys. Stat. Sol. (b) 178 (1993) 99. 
[42] G. Vörös and I. Kovács, Phil. Mag. A 72 (1995) 949.

[43] W. Günther, Zur Statik und Kinematik des Cosseratschen Kontinuums, Abh. Braunschweig. Wiss. Ges., Bd. 10 (1958) 195.

[44] H. Schaefer, Z. Ang. Math. Mech. 47 (1967) 485.

[45] G. Kluge, Wiss. Z. Techn. Hoch. Magdeburg 13 (1969) 377.

[46] M. Mişicu, Rev. Roum. Sci. Techn., Sér. méc. appl. 10 (1965) 35.

[47] C. Teodosiu, Rev. Roum. Sci. Techn., Sér. méc. appl. 10 (1965) 1461.

[48] S. Kessel, Z. Ang. Math. Mech. 50 (1970) 547.

[49] Z. Knésl and F. Semela, Int. J. Eng. Sci. 10 (1972) 83.

[50] J.P. Nowacki, Bull. Acad. Polon. Sci., Sér. sci. techn. 21 (1973) 585.

[51] N. Nowacki, Arch. Mech. 26 (1974) 3.

[52] S. Minagawa, Appl. Eng. Sci. Lett. 5 (1977) 85.

[53] D.G.B. Edelen, Int. J. Engng. Sci. 27 (1989) 653.

[54] K. Kondo, On the Geometrical and Physical Foundations of the Theory of Yielding, in Proceedings of the 2nd Japan National Congress for Applied Mechanics, Tokyo (1952) p. 41.

[55] B.A. Bilby, R. Bullough and E. Smith, Proc. Roy. Soc. (London) A 231 (1955) 263.

[56] E. Kröner and A. Seeger, Arch. Rat. Mech. Anal. 3 (1959) 97.

[57] E. Kröner, Arch. Rat. Mech. Anal. 4 (1960) 273.

[58] F.W. Hehl, J.D. McCrea, E.W. Mielke and Y. Ne'eman, Phys. Rep. 258 (1995) 1.

[59] J.F. Nye, Acta Met. 1 (1953) 153.

[60] R. deWit, Linear Theory of Static Disclinations, in Fundamental Aspects of Dislocation Theory, Vol. 1, Eds. J.A. Simmons, R. deWit and R. Bullough, Nat. Bur. Stand. (U.S.), Spec. Publ. 317 (1970) p. 651.

[61] R. deWit, J. Res. Nat. Bur. Standards 77A (1973) 49.

[62] R.D. Mindlin, Int. J. Solids Struct. 1 (1965) 265.

[63] G.A. Maugin, Material Inhomogeneities in Elasticity, Chapman and Hall, London (1993).

[64] R.A. Toupin, Arch. Rat. Mech. Anal. 17 (1964) 85.

[65] W.L. Bond and J. Andrus, Phys. Rev. 101 (1956) 1211.

[66] R. Bullough, Phys. Rev. 110 (1958) 620.

[67] V.I. Nikitenko and L.M. Dedukh, Phys. Stat. Sol. (a) 3 (1970) 383.

[68] A. Timpe, Zeits. f. Math. Phys. 52 (1905) 348.

[69] W.F. Brown, Phys. Rev. 60 (1941) 139. 
[70] J.S. Koehler, Phys. Rev. 60 (1941) 397.

[71] G. Leibfried and K. Lücke, Z. Phys. 126 (1949) 450.

[72] E. Kröner, Kontinuumstheorie der Versetzungen und Eigenspannungen, Erg. Angew. Math. 5 (1958) p. 1.

[73] E. Kröner, Continuum Theory of Defects, in: Physics of Defects (Les Houches, Session 35), R. Balian et al., eds., North-Holland, Amsterdam (1981) p. 215.

[74] R. deWit, J. Res. Nat. Bur. Standards 77A (1973) 607.

[75] W.T. Read, Dislocations in Crystals, McGraw Hill, New York (1953).

[76] C. Teodosiu, Elastic Models of Crystal Defects, Springer-Verlag, Berlin (1982).

[77] F.W. Hehl and E. Kröner, Z. Naturforschg. 20a (1965) 336.

[78] E. Kröner, Int. J. Engng. Sci. 1 (1963) 261.

[79] E. Kröner and B.K. Datta, Z. Phys. 196 (1966) 203.

[80] E. Kröner, Int. J. Solids Struct. 3 (1967) 731. 